\title{
A note from the publisher: Tables
}

\author{
The Publication Manual of the American Psychological Association (second edition) gives excellent \\ instructions for the use and proper typing style of tables on pages 43-50. Refer to these pages for \\ initial guidelines. The following will explain Psychonomic Society departures from APA style \\ and emphasize areas that are of particular importance.
}

Overall

(1) Make sure the table is necessary. Small tables with few entries can often be dealt with just as effectively in a line or two of text.

(2) Double space all tables (this is easier to read and to edit).

(3) Number all tables in the order that they are mentioned in the text. Make sure all tables are mentioned in the text.

Departures From APA Style

(1) Footnotes-Psychonomic style does not follow APA style in the use of footnotes. We have two levels of footnotes:

(a) General (denoted by "Note- . . " in italics)Qualifies, explains, or provides information relating to the table as a whole or to a major section of the table (e.g., a column or a group of columns).

(b) Specific (denoted by *,**, $\dagger,+\dagger$, also in italics)-Relates to individual entry or gives probability level (table should not require more than four levels of specific footnotes).

(c) Psychonomic journals do not use superscripted footnotes (raised $a, b, c$, etc.).

(2) All major words of table titles and column headings should be capitalized.

(3) Do not put column entries in parentheses-Label separate columns instead.

(4) Use only horizontal rules-Vertical rules are not used in Psychonomic journals. Extra space is not normally used between groups of columns (horizontal rules above groups of columns generally circumvent the need for extra space or vertical rules between columns or groups of columns).

Make the Table as Easy as Possible to Typeset

Even straightforward tables are time-consuming and difficult to typeset. You will get the best results if you take your own steps to make things easier for the typesetter.

(1) Keep the material to be typeset as simple and straightforward as possible.

(a) Avoid using symbols and typefaces that require font changes, such as italics, boldface, and mathematical symbols (e.g., $<, \pm$ ) in the body of the table. These can usually be avoided by changes in column headings and addition of footnotes.

(b) Avoid using material in tables that is impossible to typeset (e.g., Chinese characters, large circles around letters, lines connecting numbers in columns). Such material should normally be placed in figures, which are reproduced photographically.

(c) Do not mark material to be underlined.

(2) Avoid unnecessary repetitions throughout the table.

(a) Columns with the same numerical entries throughout the table or throughout sections of the table can be put in footnotes (e.g., "In Condition $1, n=20$ for each group; in Condition 2 , $\mathrm{n}=30$ for each group"); repetitious verbal entries can usually be put in headings or in footnotes (e.g., "RTs are given in milliseconds").

(b) Delete superfluous zeros before decimal points.

(3) Keep the table format as simple and straightforward as possible.

(a) Arrange tables so that similar numbers fall into columns (i.e., do not mix $F$ values with numbers such as 00.00 , dfs with numbers such as 00,00 , and MSes with numbers such as .0000 in one column; make columns of $00.00 \mathrm{~s}, 00,00 \mathrm{~s}$, and .0000s).

(b) Do not combine two tables of dissimilar format into one table (e.g., if Sections $A$ and $B$ of Table 1 are not of similar format, Section $A$ should be Table 1 and Section B, Table 2).

(c) Do combine small tables of identical format with few entries (e.g., rather than four tables of four columns and one row giving data for each of four experiments, provide one table with four columns and four rows, one for each experiment).

Make the Table Intelligible for the Typesetter

The most important thing to remember regarding the typing of tables in manuscripts is that, although the people who will read your paper after it is published will, for the most part, be scientists (predominately PhDs and graduate students in psychology), the people who will typeset your paper before it is published will, for the most part, not be. Don't assume everything that you understand is inherently understandable.

Furthermore, due to the division of duties in the Publications Office, the tables will often be the only part of your manuscript that the person who typesets them will see. Make sure each table is self explanatory, even to someone who has not read your paper.

(1) Define (or avoid using) all abbreviations.

(2) Define measurements used for values in the table (e.g., "RTs are given in milliseconds; auditory thresholds are given in decibels").

(3) Label all columns clearly.

(a) Column headings should be understandable enough that they can be rewritten by nonscientific personnel (e.g., if space prohibits the use of your preferred headings).

(b) Column headings should be centered over the columns to which they apply.

(c) Avoid using the body of the table to imply column headings (e.g., $1.00 \pm .01$ in body to imply "Mean" over Column 1 and "SE" over Column 2).

(4) Avoid using asterisks and daggers to denote anything other than footnotes pertaining to particular entries in the body of the table. Define all asterisks and daggers used (in table footnotes, not in the text). 